\title{
EVALUATION OF PCOS IN OBESE AND NON-OBESE PATIENTS USING TRANSVAGINAL OVARIAN AND UTERINE ARTERY COLOR DOPPLER
}

\section{Dr. (Prof.) Alka Agrawal}

\section{Dr. Vivek Yonati*}

\section{Dr. Gaurav} Bhandari

\section{Dr Ark Sarkar}

\section{Dr Prakhar Nigam}

\section{Professor \& HOD, Department Of Radiodiagnosis, M.G.M. Medical College} \& M.Y. Hospital, Indore.

Associate Professor, Department Of Radiodiagnosis, M.G.M. Medical College \& M.Y. Hospital, Indore. ${ }^{*}$ Corresponding Author

Assistant Professor, Department Of Radiodiagnosis, M.G.M. Medical College \& M.Y. Hospital, Indore.

Third Year P.G. Resident, Department Of Radiodiagnosis, M.G.M. Medical College \& M.Y. Hospital, Indore.

Second Year P.G. Resident, Department Of Radiodiagnosis, M.G.M. Medical College \& M.Y. Hospital, Indore.

ABSTRACT Background: Polycystic ovarian syndrome (PCOS) is a common endocrine disorder of premenopausal women affecting approximately $5-10 \%$ of female population in their reproductive years ${ }^{(1)}$. The clinical, biochemical as well as the radiological spectrum of PCOS and their correlations have gained importance over the years owing to complex pathophysiology of this syndrome, which still remains partially understood. Aims \& Objectives: To study hemodynamic changes in ovarian and uterine arteries in PCOS patients using color doppler ultrasound, along with their clinical and biochemical parameters. Methods: A total of 60 patients with clinical history of infertility (30 obese \& 30 non-obese) and 30 control (fertile women) were taken, who underwent transvaginal ultrasound and color doppler scan, and were correlated clinically and hormonally. Results: The ovarian artery mean pulsatility index (PI) was observed to be 4.05 in control group and 2.58 among PCOS cases, which is significantly decreased in cases. Comparing non-obese and obese PCOS cases, the mean ovarian artery PI was found to be 2.65 and 2.51 respectively. The difference was not significant. In our study, the resistive index (RI) of ovarian artery also showed a significant decrease among PCOS cases with mean value of 0.62, compared to 0.9 in the control group. The PI and RI of uterine artery in both obese and non-obese cases were significantly increased in comparison to control group. Conclusion: Transvaginal colour doppler helps in adequate evaluation of haemodynamic changes in small vessels of utero-ovarian circulation. It can aid in diagnosis as well as serve as prognostic indicator in patients suffering from PCOS.

\section{KEYWORDS : PCOS, Ultrasonography, PI, RI}

\section{INTRODUCTION}

PCOS is a diverse pathological condition characterized by reproductive disorders (oligomenorrhea/amenorrhea) which is frequently associated with obesity, hirsutism, acne vulgaris, hyperandrogenism and insulin resistance ${ }^{(2)}$. Polycystic ovarian syndrome (PCOS) is the most common cause of anovulatory infertility accounting for $>70 \%$ of $\operatorname{cases}^{(3)}$.

Ultrasound has been a modality of choice for identification of polycystic ovarian morphology (PCOM), which was standardised back in 2003, in the form of Rotterdam's sonographic criteria defined as an ovary with 12 or more follicles measuring $2-9 \mathrm{~mm}$ and/or increased ovarian volume $\left(>10 \mathrm{~cm}^{3}\right)^{(4)}$. Ultrasound assessment of ovarian morphology is considered to be necessary in the diagnosis of PCOS and gold standard for defining PCOM ${ }^{(5)}$. Transvaginal ultrasound is more sensitive than transabdominal ultrasound for visualization of the internal structure of the ovary, particularly in obese patients.

It has been established that hemodynamics of ovarian and uterine arteries in PCOS patient differ from that of a normal woman ${ }^{(6)}$. However, no consensus with respect to change in vascular pattern and their doppler characteristics have been achieved so far.

In our study, our aim was to evaluate the inherent changes in vascular blood flow patterns of uterine and ovarian arteries in PCOS patients using color and pulsed doppler as a tool and to assess their relationship with clinical and endocrine findings. Doppler analysis of ovarian and uterine arteries in PCOS may provide further insight about the pathophysiology of the syndrome and will help to formulate an improvised approach towards the treatment.

\section{MATERIAL AND METHODS}

A total of 60 patients with clinically suspected polycystic ovarian syndrome and 30 healthy fertile women were evaluated in the Department of Radiodiagnosis, M.G.M. Medical College and M.Y. Hospital, Indore from May 2019 to October 2020 after written informed consent.

The patients were subdivided into 3 groups:

Group I- 30 Healthy fertile women

Group II- 30 Non-obese women diagnosed as PCOS

Group III- 30 Obese women diagnosed as PCOS

\section{RESULTS}

The mean age in group I, group II and group III were 28.43 years $[S D=4.24], 28.37$ years $\quad[S D=3.28]$ and 28.10 years [SD $=3.82]$. The mean age of the three groups did not differ significantly.

Among PCOS cases in our study, 50\% had complaints of acne, $32 \%$ had hirsutism and $52 \%$ had menstrual irregularities. In our study, when compared between non-obese and obese women with PCOS, infertility was seen in $87 \%$ and $93 \%$ respectively. Primary infertility was seen in $53 \%$ of non-obese women with PCOS and $58 \%$ of obese women with PCOS. Secondary infertility was seen in $32 \%$ of non-obese women with PCOS and $34 \%$ of obese women with PCOS. 
Table: 1 - Hormonal and ultrasound findings among the three studied groups.

\begin{tabular}{|l|l|l|l|}
\hline VARIABLES & GROUP I & GROUP II & GROUP III \\
\hline LH $^{*}$ (in mIU/mL) & $9 \pm 2.1$ & $14.78 \pm 2.38$ & $17.23 \pm 3.22$ \\
\hline FSH ${ }^{\# \# ~(i n ~ m I U / m L) ~}$ & $7.02 \pm 1.42$ & $6.57 \pm 1.08$ & $7.08 \pm 1.62$ \\
\hline LH/FSH & $1.30 \pm 0.25$ & $2.26 \pm 0.24$ & $2.46 \pm 0.28$ \\
\hline $\begin{array}{l}\text { MEAN OVARIAN } \\
\text { VOLUME (In ml) }\end{array}$ & 5 & $10.56 \pm 1.5$ & $14.2 \pm 1.8$ \\
\hline $\begin{array}{l}\text { NO. OF OVARIAN } \\
\text { FOLLICLES }\end{array}$ & $5 \pm 1$ & $13 \pm 2$ & $14 \pm 2$ \\
\hline $\begin{array}{l}\text { OVARIAN ARTERY } \\
\text { PULSATILITY INDEX }\end{array}$ & $4.05 \pm 0.56$ & $2.65 \pm 0.38$ & $2.51 \pm 0.38$ \\
\hline $\begin{array}{l}\text { OVARIAN ARTERY } \\
\text { RESISTANCE INDEX }\end{array}$ & $0.89 \pm 0.05$ & $0.61 \pm 0.07$ & $0.64 \pm 0.07$ \\
\hline $\begin{array}{l}\text { UTERINE ARTERY } \\
\text { PULSATILITY INDEX }\end{array}$ & $1.65 \pm 0.44$ & $4.43 \pm 0.89$ & $6.64 \pm 1.02$ \\
\hline $\begin{array}{l}\text { UTERINE ARTERY } \\
\text { RESISTANCE INDEX }\end{array}$ & $0.78 \pm 0.15$ & $0.91 \pm 0.07$ & $0.88 \pm 0.10$ \\
\hline
\end{tabular}

${ }^{\#}$ Luteinizing hormone ${ }^{\# \#}$ Follicle stimulating hormone

Significant increase in mean LH values and LH/FSH ratio was noted in cases, as compared to control group. There was significant increase in ovarian volumes among cases than in controls ( $\mathrm{P}$ value $<0.0001$ ). Significant increase in number of small ovarian follicles was observed in cases as compared to controls.

There was statistically significant decrease in ovarian artery pulsatility index (PI) and resistance index (RI) in patients with PCOS than in control women ( $\mathrm{P}$ value $<0.0001$ ). There was statistically insignificant difference between ovarian doppler indices in normal and overweight cases (Pvalue $>0.05$ ).

There was statistically significant increase in uterine artery PI and RI in patients with PCOS than in control women (P value < 0.0001 ). Statistically significant increase in PI of Uterine artery was found in obese cases than non-obese cases ( $\mathrm{P}$ value $<$ $0.0001)$. However, increase in uterine artery RI in obese cases as compared to non-obese cases was not significant.

\section{DISCUSSION}

PCOS is a heterogenous disorder with convergence of multisystem endocrine derangements and has emerged as one of the leading health issues among women as it affects her fertility. Ultrasonography helps in early and precise diagnosis of PCOS.

The mean age was 28.37 years in non-obese and 28.10 years in obese patients representing the mean age group to be diagnosed with PCOS. The presence of obesity in cases (Group III) was associated with significantly higher clinical complains of acne, hirsutism and menstrual irregularities as compared to non-obese cases (Group II). This reemphasizes the positive correlation between body mass index (BMI) and features of hyperandrogenism in PCOS patients, along with the importance of weight reduction and lifestyle modifications.

The biochemical results of our study were in agreement with previous studies ${ }^{(7,8)}$ demonstrating that women suffering from PCOS have significantly increased serum LH values and increased LH/FSH ratio.

On B-mode ultrasound study, mean ovarian volume was found to be lower in control group $(5 \mathrm{ml}$ ) as compared to PCOS cases $(12.38 \mathrm{ml})$. All PCOS cases in our study showed $>12$ ovarian follicles of size 2 to $9 \mathrm{~mm}$, which are in concordance with PCO morphology.

Pulsatility index and resistivity index are used to evaluate resistance in an arterial system. In our study, the ovarian artery mean PI and RI was lower in cases (2.58 and 0.62 respectively) as compared to controls (4.05 and 0.9 respectively). There was no significant difference in PI and RI of right and left ovarian artery in both PCOS cases and control group. The vascular impedance in the ovarian artery has been associated with phenomenon of massive angiogenesis, influenced by LH in unstimulated cycles or by human chorionic gonadotrophin in stimulated cycles. The increased serum concentrations of oestrogens cause vasodilatation in the ovarian artery, and consequently, a decrease in vascular impedance $^{(9)}$

In our study, the mean PI and RI of uterine artery was found to be significantly higher (5.53 and 0.89 respectively) among PCOS cases than in control group ( 1.65 and 0.78 respectively). Yedla M Mala et $\mathrm{al}^{(10)}$ in their study showed a significant rise in PI and RI among PCOS cases.

Androgens, characteristically DHEAS, have been shown to exert a direct vasoconstrictive effect on vascular tissues by acting via specific receptors located on the arterial wall. Hyperinsulinemia and obesity are closely linked, and this in turn lead to hyperandrogenism. However, in the study done by Adams et al ${ }^{(11)}$ among normal women with PCO morphology, showed ovarian stromal cells are the primary source of hyperandrogenism, which explains higher PI and RI values among non-obese cases in our study.

Other than oligomenorrhea, the increased resistance in uterine vessels explains difficulty in conception among PCOS patients. A. El-Mazny et $\alpha{ }^{(12)}$ in their study found that uterine artery PI and RI were significantly increased in the unexplained infertility group leading to impaired endometrial perfusion and implantation.

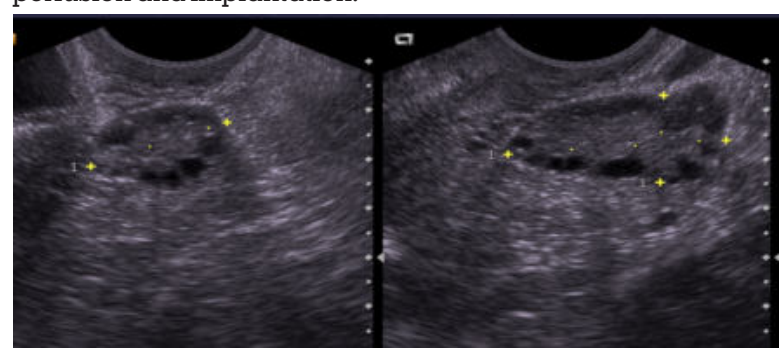

Figure 1-Polycystic ovary with peripherally arranged follicles

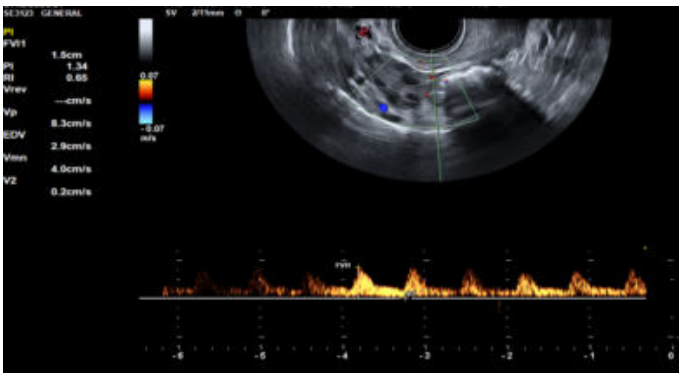

Figure 2- Ovarian artery doppler in PCO case with low resistance flow (low RI \& PI)

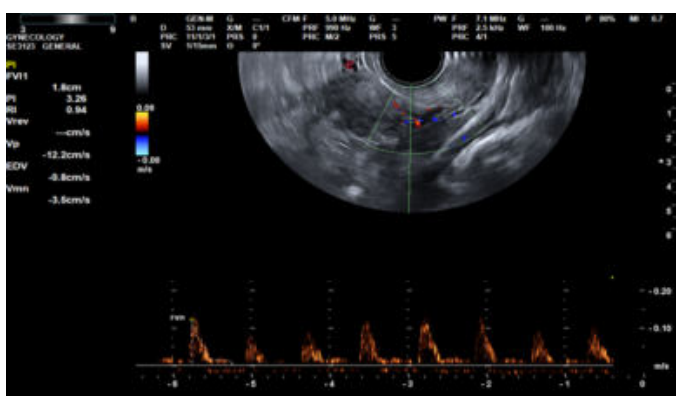

Figure 3- Uterine artery doppler in a PCOS case with high resistance flow (raised RI \& PI) 
CONCLUSION

PCOS cases in our study showed significant changes in blood flow pattern in uterine and ovarian arteries. We conclude that PCOS is a syndrome of high uterine vascular resistance and increased ovarian stromal blood flow.

Though not part of Rotterdam criteria, transvaginal colour doppler helps in the evaluation of this haemodynamic changes in small vessels of utero-ovarian circulation. Being an easy tool, we recommend incorporation of doppler ultrasound as a part of routine ultrasound scan, in patients with PCOS and in patients of $\mathrm{PCO}$ morphology without features of hyperandrogenism.

\section{REFERENCES}

1. Hart R. Definition, prevalence \& symptoms of polycystic ovaries \& polycystic ovarian syndrome. In Allahbadia G, Agrawal R, editors. Polycystic ovarian syndrome. lt $^{\text {st }}$ ed.UK: Anshan Limited ;2007.P.15-26.2.Taylor AE 1998 Polycystic ovary syndrome. Endocrinol Metab Clin North Am 27:877-902

3. Parsanezhad ME, Bagheri MH, Alborzi S, et al. Ovarian stromal blood flow changes after laparoscopic ovarian cauterization in women with polycystic ovary syndrome. Hum Reprod 2003 Jul; 18(7):1432e7.

4. Balen A H. What is new in polycystic ovarian syndrome.In:Bonnar J,DunlopW,editors.Recent advances in obstetrics and gynecology.2nd ed.UK:Royal society of Medicine Press ;2005.P.147-58

5. Balen AH, Laven JS, Tan SL, Dewailly D. Ultrasound assessment of the polycystic ovary: International consensus definitions. Hum Reprod Update 2003;9:505-14.

6. Aleem FA, Predanic M. Transvaginal color Doppler determination of the ovarian and uterine blood flow characteristics in polycystic ovary syndrome. Fertil Steril 1996;65:510.

7. Ajossa S, Guerriero S, Paoletti AM, et al. Uterine perfusion and hormonal pattern in patients with polycystic ovary syndrome. J Assist Reprod Genet 2001;18:436.

8. Adams J, Franks S, Polson DW, Mason HD, Abdulwahid N, Tucker M, et al. Multifollicular ovaries: Clinical and endocrine features and response to pulsatile gonadotropin releasing hormone. Lancet 1985;2:1375-9.

9. Salmi DJ, Zisser HC, Jovanovic L. Screening for and treatment of polycystic ovary syndrome in teenagers. Exp Biol Med (Maywood) 2004 May:229(5):369e77

10. Yedla M Mala, Sharda Ghosh, Reva Tripathi. Three-dimensional power Doppler imaging in the diagnosis of polycystic ovary syndrome, Ultrasound Obstet Gynecol 2005; 26(4):341.

11. Judith M. Adams, Ann E. Taylor, William F. Crowley and Janet E. Hall. Polycystic Ovarian Morphology with Regular Ovulatory Cycles: Insights into the Pathophysiology of Polycystic Ovarian Syndrome. The Journal of Clinical Endocrinology \& Metabolism 89(9):4343-4350

12. Akmal El-Mazny, Nermeen Abou-Salem, Hossam ElShenoufy. Doppler study of uterine hemodynamics in women with unexplained infertility. European Journal of Obstetrics \& Gynecology and Reproductive Biology 1712013 (8487) 